\section{Digital and cephalic vasomotor responses as a function of intensity of electric shock*}

\author{
H. D. KIMMEL and F. R. TERRANT \\ University of South Florida, Tampa, Fla. 33620
}

To evaluate predictions from Sokolov's position regarding the orienting and defensive reflexes, $42 \mathrm{Ss}$ were given 50 shocks at $0.5,1.0,2.0,4.0$, or $8.0 \mathrm{~mA}$. The finding that vasomotor orienting responses increased in frequency as a function of shock intensity, while defensive responses did not, was taken as evidence contrary to Sokolov's theory.

Sokolov (1963) differentiated the orienting reflex (OR) from the defensive reflex (DR) on the basis of the dilation or constriction, respectively, of cephalic blood vessels and the concurrent constriction of digital vessels. Cephalic dilation a c companied by digital vasoconstriction is taken as representing an $\mathrm{OR}$, while cephalic constriction with concurrent digital constrictions represents a DR. In an effort to elucidate further the properties of these measures, several recent studies have investigated the changes of cephalic blood content (BC) produced by stimuli of graded intensities, the assumption being that low-intensity stimuli should produce cephalic dilation (i.e., ORs), while high intensities should produce cephalic constriction (i.e., DRs). On the basis of their findings, Raskin, Kotses, and Bever conciuded that ". . . changes in forehead BC do not provide an adequate index for differentiating ORs from DRs [1969a]," and that "... changes in BC provide a misleading conciusion about the type of reflex evoked [1969b]." These results were consistent with earlier findings of Kimmel, Pendergrass, \& Kimmel (1968), that cephalic responses were too unreliable for use in identifying ORs.

Although Sokolov's approach to defining ORs and DRs involves simultaneous measurement of both cephalic and digital vasomotor responses, the studies mentioned above measured only cephalic BC changes. Thus, it was felt that further evaluation of Sokolov's position regarding the discrete category of responses evoked by stimuli of different intensities should involve measures at both loci. The advantage of employing both measures is that cephalic BC changes which are not

*This research was done at Ohio University under USPHS Grant MH-12262-04. Paul Gade, Thomas Reynolds, Nolan Russell, and Robert Yaremko assisted in data collection and reduction. padded office-type chair. DC shocks from an Argonaut stimulator were delivered to the volar surface of $\mathrm{S} s$ right forearm through electrodes composed of $3 / 4$-in. zinc disks coated with a light layer of saline electrode paste and held $1 / 2$ in. apart by a rubber strap. The skin on the arm was first cleaned with acetone and briskly rubbed until slightly red. The cephalic photoplethysmograph was placed on S's left temple between the hairline and the orbital bone and was held in place by a rubber head band. The digital plethysmograph was contained in a spring-loaded ring and was placed over the fingernail of the left index finger, which was further covered by a small black cloth bag. The plethysmographic signals were amplified and recorded on a Grass polygraph (5P1 preamplifier).

After placement of the shock electrodes and recording devices, $S$ was seated in the IAC chamber and the door was closed, leaving $S$ in total darkness. The Ss were then reminded via an intercom that the purpose of the experiment was to study the effects of mild, brief electric shocks on various physiological reactions and that their task was merely to pay attention to the stimuli, to remain alert, and to maintain a relaxed and relatively motionless position.

Following a 5 -min rest period, each $S$ received a series of 50 shocks having an interstimulus interval of 20 to $60 \mathrm{sec}$ with a mean of $40 \mathrm{sec}$. Shock intensities were $0.5,1.0,2.0,4.0$, and $8.0 \mathrm{~mA}$ for five independent, randomly assigned groups of $8,9,10$,

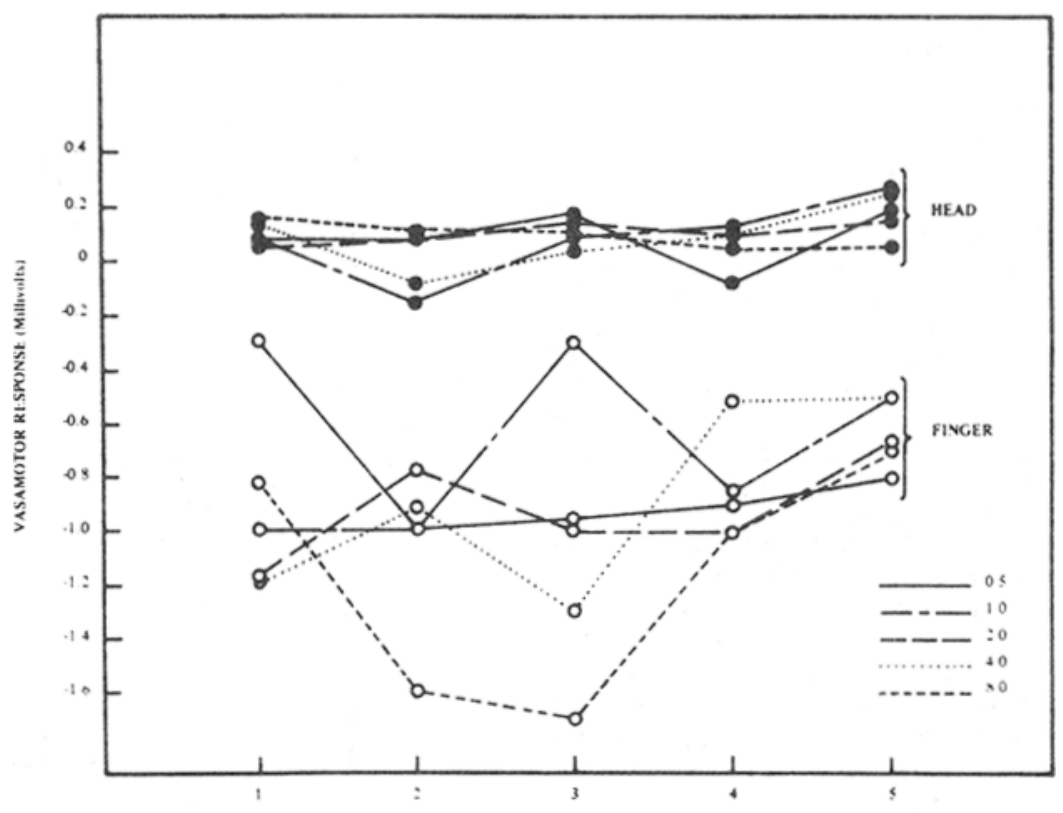

BLOCKS OF TEN TRIALS

Fig. 1. Mean digital and cephalic vasomotor responses (in millivolts) during five blocks of 10 trials each, at five shock intensities. 


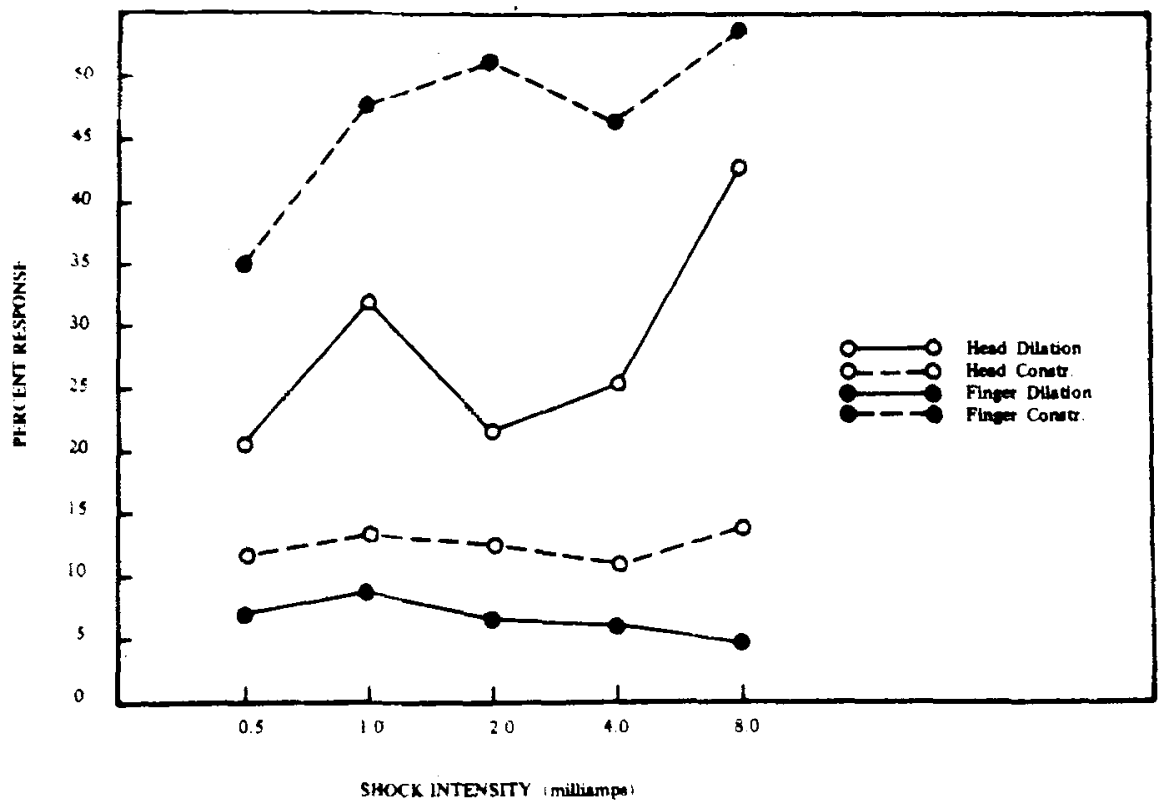

Fig. 2. Mean percentages of head and finger dilations or constrictions as a function of shock intensity.

\section{8 . and 7 Ss. respectively.}

\section{RESLLTS}

Figure 1 presents the mean change (in millivolts) of digital and cephalic $B C$ in each block of 10 trials for each shock intensity group. Blood volume changes were measured as the maximum of the initial deviation from preresponse level of traced diastolic volumes for responses beginning 2 to $8 \mathrm{sec}$ from shock onset. The criterion for the beginning of a response was two or more successive diastolic volumes which deviated from the immediately preceding diastolic volume by an amount exceeding the amplitude of the preceding pulses. When no volume change in a given trial met this criterion, the recorded response magnitude was zero. The preresponse volume was the diastolic volume immediately preceding the criterion change. In general, cephalic $\mathrm{BC}$ changed very little for all trial blocks and intensities. Digital BC exhibited noticeable decreases (constriction) with an unsystematic pattern over trial blocks.

Analysis of variance revealed that postshock $\mathrm{BC}$ changes measured at the finger were significantly different from those measured at the head (i.e., tended more toward constriction), $F(1.37)=64.19, p<.001$, and that the overall effect of trial blocks was also significant, $F(4,148)=2.42$, $\mathrm{p}<.05$. No other effects approached significance.

Figure 2 presents percentages of dilation and constriction at the head and finger for each of the five intensities. Dilations were criterion-level increases of diastolic shock intensity. dilations and finger constrictions showed increased percentages as shock intensity increased, the latter being greater in frequency than the former. Analysis of variance showed that the effect of locus of measurement was significant $(p<.01)$, as was type of response $(p<.001)$. The Response (dilation-constriction) by Locus interaction was also highly significant $(\mathrm{p}<.001)$, since the difference between loci depended greatly upon which type of response was considered. The intensity variable did not show a significant effect.

Figure 3 shows the percentage of "orienting" and "defensive" reflexes occurring at each of the five intensities. The percentages were obtained by using Sokolov's definition of ORs and DRs, i.e., disparate or conjoint head constriction-dilation with finger constriction. Concomitant cephalic dilations and digital constrictions defined orienting reflexes, while concomitant cephalic constrictions and digital constrictions defined defensive reflexes. Cephalic changes not accompanied by digital constrictions were counted as nonresponses, For all intensities, the percentage of orienting responses was greater than that for defensive reflexes, the difference in percentages for the two types of response tending to increase as intensity increased. The percentage of ORs was significantly greater than that for DRs $(p<.01)$,

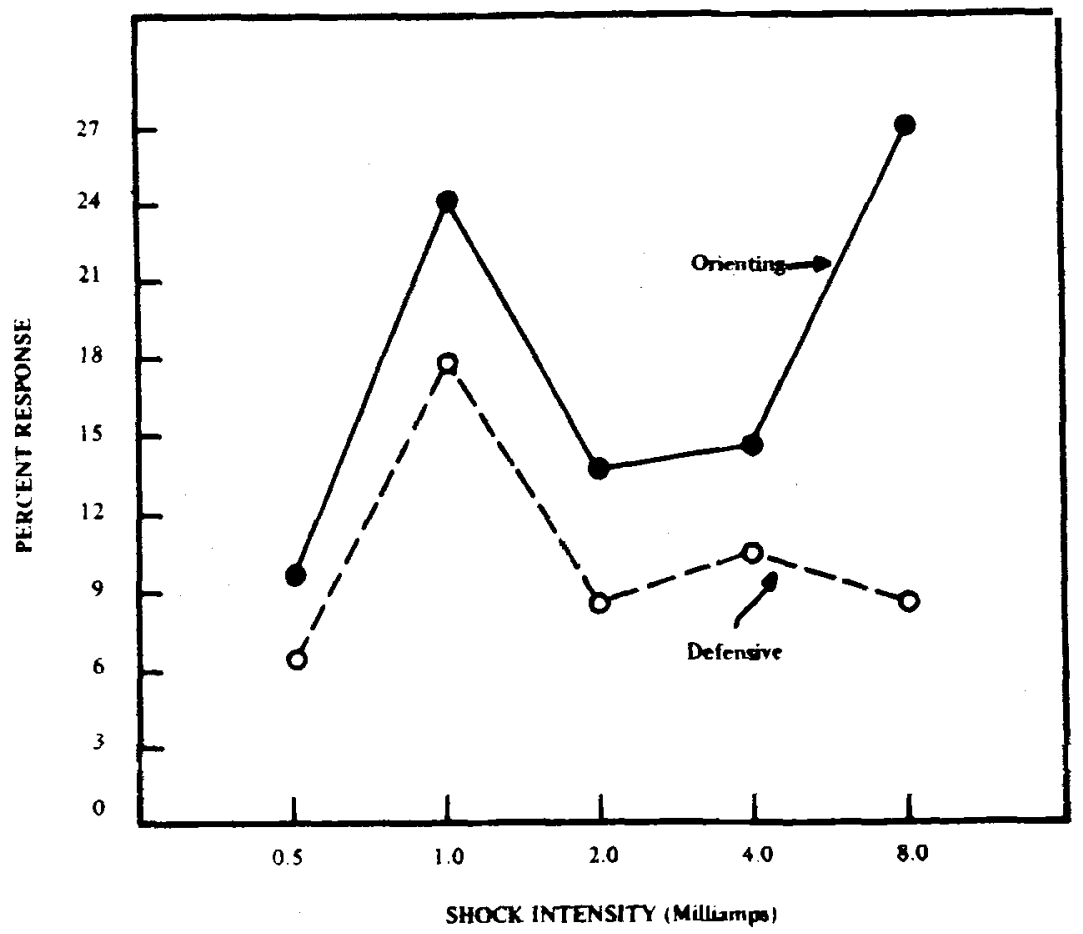

Fig. 3. Mean percentages of orienting and defensive responses as a function of 
and the Response (OR vs DR) by Intensity interaction was significant $(\mathrm{p}<.05)$.

The interaction seems to have been due primarily to the large difference between ORs and DRs at the $8.0-\mathrm{mA}$ intensity.

\section{DISCUSSION}

The present findings support the generalization that the dominant vascular response to shock is an increase in cephalic blood volume and a decrease in digital blood volume, the magnitude of the change being greater for the latter than for the former. Further, the frequency of changes increases with shock intensity, leading to an increase in frequency of ORs, as defined by Sokolov (1963), but to no change in DR frequency.

These findings are clearly contradictory to the findings predicted by Sokolov (1963). Sokolov's theory predicts an increased frequency of DRs with increased shock intensity and a reduction of $O R$ frequency. The results of Fig. 3 are not in agreement with this contention. Since shocks at $8.0 \mathrm{~mA}$ were definitely noxious, it may not be argued that the range of shock intensities is not sufficiently high to permit DRs to be revealed.

Raskin et al (1969b), who showed that cephalic $\mathrm{BC}$ increases and pulse amplitude (PA) decreases in response to tones, have argued persuasively that the cephalic vasomotor response to stimulation is actually vasoconstriction. This argument was based on their demonstration that increased $\mathrm{BC}$ at the head is highly correlated with increased heart rate and is not due to vasodilation. Taken in conjunction with the present data, Raskin et al's findings suggest that the vasomotor response to stimulation is universally constriction, regardless of the locus of measurement. Hence, for purposes of further research, it appears that digital plethysmographic measures of constriction via pulse amplitude would suffice as indicants of vasomotor activity and would minimize contamination from heart-rate changes. Since adequate placement and securing of the photoelectric transducer on the forehead is very difficult to attain, there would be an additional practical benefit of a shift to digital research in the future.

\section{REFERENCES}

KIMMEL, H. D., PENDERGRASS, V. E., \& KIMMEL, E. B. Modifying children's orienting reactions instrumentally. Conditional Reflex, 1967, 2, 227-235.

RASKIN, D. C., KOTSES, H., \& BEVER, J. Autonomic indicators of orienting and defensive reflexes. Journal of Experimental Psychology, 1969a, 80, 423-433.

RASKIN, D. C., KOTSES, H., \& BEVER, J. Cephalic vasomotor and heart rate measures of orienting and defensive reflexes. Psychophysiology, 1969b, 6, 149-159.

SOKOLOV, $\mathrm{E}$. N. Perception and the conditioned reflex. New York: Macmillan, 1963. 